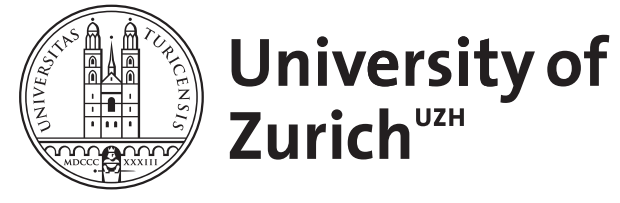

Zurich Open Repository and Archive

University of Zurich

University Library

Strickhofstrasse 39

CH-8057 Zurich

www.zora.uzh.ch

Year: 2011

\title{
Schwerpunkt: Hermann Cohens Philosophie
}

Renz, Ursula

DOI: https://doi.org/10.1524/dzph.2011.0017

Posted at the Zurich Open Repository and Archive, University of Zurich

ZORA URL: https://doi.org/10.5167/uzh-52936

Journal Article

Published Version

Originally published at:

Renz, Ursula (2011). Schwerpunkt: Hermann Cohens Philosophie. Deutsche Zeitschrift für Philosophie, $59(2): 221-226$.

DOI: https://doi.org/10.1524/dzph.2011.0017 


\section{SCHWERPUNKT: HERMANN COHENS PHILOSOPHIE}

Hermann Cohen gehörte zweifelsohne zu den wichtigsten deutschen Philosophen vor und nach der Wende zum 20. Jahrhundert. Mit seinem Namen verbindet sich noch heute der Name seiner Lehrstätte Marburg, der er zu internationaler Berühmtheit verholfen hat. Bekannt ist Cohen auch als einer der Begründer des Neukantianismus. Doch über dessen Fortentwicklung zu einer eigenständigen systematischen Philosophie wissen selbst Philosophen wenig.

Wer war Hermann Cohen, und was zeichnete ihn und sein Denken aus? Hermann Cohen (4. Juli 1842-4. April 1918) war Philosoph durch und durch. Zeit seines Lebens ist er mit einer Konsequenz sondergleichen für die Philosophie eingetreten. Der Philosophie galt seine Liebe, und diese Liebe bestimmte über weite Strecken sein Verhältnis zur Wissenschaft und zur Kunst, zu den Ansätzen anderer Philosophen, zur philosophischen Tradition und sogar - wenn auch nicht ausschließlich - zur Religion. Um der Philosophie willen hat er 1861 das Breslauer Rabbinerseminar, in das er 1857 eingetreten war, vorzeitig verlassen. ${ }^{1}$ Um der Philosophie willen distanzierte er sich Ende der 60er Jahre des 19. Jahrhunderts von seinen von der Völkerpsychologie Hermann Steinthals und Moritz Lazarus' geprägten philosophischen Anfängen und wandte sich Kants kritischer Philosophie zu, dessen Auslegung er neuen philologischen Maßstäben unterwarf. ${ }^{2}$ Um der Philosophie willen unterwarf er ab 1883 Kants

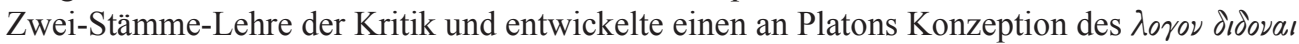
orientierte Auffassung von kritischem Idealismus. Noch seine Marburger Abschiedsvorlesung vom 1. August 1912 schloss er mit der Aufforderung: „Behalten Sie für Ihr Leben die Philosophie lieb! Behalten Sie die Philosophie lieb! Lieb! Ja, wahrhaftig lieb! ${ }^{* 3}$

Als Philosophie ließ Cohen indes längst nicht alles gelten, was unter diesem Namen kursierte. Seit seiner Auseinandersetzung mit Kant maß Cohen sämtliche philosophische Unterfangen an einem idealen Begriff des kritischen Idealismus, der keineswegs in allen Punkten identisch mit Kants Ansatz war. Wo der kritische Idealismus ins Hintertreffen geriet, da sah Cohen die Philosophie in der Gefahr, in Vorurteile oder in den Mythos zurückzufallen. Solche Gefahr drohte nach Cohen im Bereich der theoretischen Philosophie vor allem jeglichem Psychologismus ${ }^{4}$; in der Ethik materialistischen oder naturalistischen Tendenzen ${ }^{5}$ und in der

1 Die wissenschaftliche Sozialisation, die Cohen im Breslauer Rabbiner-Seminar erfahren hat, zeichnet der Beitrag von Hartwig Wiedebach in diesem Heft nach.

2 Welche Bedeutung Cohen der Wiederentdeckung von Kant für die Philosophie zumaß, geht aus der Vorrede zur ersten Auflage von Kants Theorie der Erfahrung sehr klar hervor; siehe H. Cohen, Werke, 1.3, hg. vom Hermann-Cohen-Archiv unter der Leitung von H. Holzhey, Hildesheim 1977 ff. (im Folgenden zitiert als Werke, plus Band, plus Seitenzahl).

3 Hinrich Knittermeyer hat diese Äußerung am selben Tag in einem Brief an seinen Vater festgehalten; siehe H. Holzhey, Hermann Cohens Weggang aus Marburg, in: Neue Zürcher Zeitung, 21. März 1971.

4 Siehe dazu eindrucksvoll die Entgegensetzung von kritischem Idealismus und Psychologismus, in: H. Cohen, Logik der reinen Erkenntnis, Werke, 6, $597 \mathrm{f}$.

5 Siehe dazu etwa ders., Ethik des reinen Willens, Werke, 7, 234. 
Ästhetik und Kunstphilosophie dem Ästhetizismus. ${ }^{6}$ Selbst die Phänomenologie, die - wie Cohen mit seinem kritischen Idealismus - darum bemüht war, der Philosophie angesichts der Ausdifferenzierung der Wissenschaften einen angemessenen Platz und eine eigene Aufgabe zu sichern, bedachte er mit warnenden Worten. ${ }^{7}$

Nicht frei von warnenden Untertönen sind auch Cohens Aussagen zur Philosophiegeschichte. Getreu der schon in seiner Stellungnahme Zur Kontroverse zwischen Trendelenburg und Kuno Fischer formulierten Forderung, dass der Philosophiehistoriker Philosoph sein und systematisch Anteil am Problem nehmen müsse, das er darstelle ${ }^{8}$, misst auch Cohen in seinen Exkursen zur Philosophiegeschichte die dargestellten Ansätze immer an dem, was er als das entscheidende Problem einer bestimmten Thematik ansieht. Seine philosophiegeschichtlichen Diagnosen sind selten von ausführlichen Diskussionen begleitet, doch selbst mit seinen scheinbar beiläufigen Bemerkungen trifft er oft ins Schwarze, was immer wieder zu einer systematischen Auseinandersetzung mit den kritisierten oder gewürdigten Autoren einlädt. ${ }^{9}$ Wenn er eine bestimmte Position für falsch hält, macht er das ebenso unmissverständlich wie temperamentvoll klar. ${ }^{10}$ Allerdings werden auch Gegner durchaus gewürdigt. Ob Spinoza, Hegel oder Marx - sie alle sind für Cohen ebenso Gegenstand von Hochachtung wie von radikaler Kritik. ${ }^{11}$ Und er differenziert auch meist klar zwischen der Auseinandersetzung mit den Positionen selber und Trends, die an diese anschließen. ${ }^{12}$

6 Siehe dazu vor allem auch Cohens Auseinandersetzung mit Schopenhauer in der Ästhetik des reinen Gefühls, die in der Aussage mündet: „Entweder ist die Ästhetik das Glied eines Systems der Philosophie, oder aber das System und mit ihm die Philosophie selbst wird vernichtet, wenngleich sie in Ästhetik aufgelöst wird.“ (Werke, 8, 16) Cohen hebt sich damit von dem etwas früher zitierten Gedanken Schopenhauers ab: „Gesetzt es gelänge, eine vollkommen richtige, vollständige und in das Einzelne gehende Erklärung der Musik, also eine ausführliche Wiederholung dessen, was sie ausdrückt, in Begriffen zu geben, so würde diese [...] die wahre Philosophie sein." (Zitiert in: Werke, $8,15)$

7 Siehe die Ausführungen, die Cohen dazu in einem Zusatz zur zweiten Auflage der Logik der reinen Erkenntnis von 1914 angestellt hat (Werke, 6, 55-57). Cohen sieht in der Phänomenologie eine neue Scholastik, die „günstigstenfalls Ontologie“ betreibe, „wenn sie durchaus nicht Psychologie sein" wolle und solle. Problematisch daran ist für ihn, „dass dem Inhalt der Logik ein Vorinhalt zugemutet wird. Während die Logik, sofern sie selbständig werden soll, ihren vollen Begriffsinhalt in sich selbst zu erstellen hat, soll ausdrücklich dort eine Vorarbeit geleistet und der Logik überliefert werden." (Ebd., 56) Cohen hat also die Entstehung der Husserlschen Phänomenologie zur Kenntnis genommen; inwieweit er sich auch mit deren Entwicklung und den Schriften aus der so genannten transzendentalen Phase Husserls auseinandergesetzt hat, ist offen. Man kann jedoch vermuten, dass auch diese Schriften keineswegs eine günstigere Beurteilung erfahren hätten, zumal der Gegensatz zwischen dem wissenschaftlichen Realismus Cohens und der Phänomenologie schärfer wird, wenn sich beide Ansätze unter Rückgriff auf transzendentalphilosophische Überlegungen als erste Philosophie verstehen.

8 H. Cohen, Schriften zur Philosophie und Zeitgeschichte, Bd. I, hg. v. A. Görland u. E. Cassirer, Berlin 1928, 272.

9 Es ist nicht verwunderlich, dass viele Autoren, zu denen sich Cohen affirmativ verhält - dazu gehören neben Platon und Kant vor allem auch Cusanus, Newton, Galilei und Leibniz sowie in der Ästhetik die Dichter der deutschen Klassik -, auch in Cassirers Philosophie eine zentrale Rolle spielen.

10 Siehe etwa seine Aussagen zu Aristoteles in: Logik der reinen Erkenntnis, Werke, 6, $13 \mathrm{f}$. sowie $45 \mathrm{f}$;; oder zu Hegel in: Ethik des reinen Willens, Werke, 7, $331 \mathrm{f}$.

11 Siehe zum Beispiel die Ausführungen zu Marx in: ebd., $313 \mathrm{f}$.

12 Vgl. die Ausführungen zu Spinoza in: Ethik des reinen Willens, Werke, 7, 458 f.; oder zu Rousseau in: ebd., 13. 
Cohens Gleichsetzung von Philosophie mit kritischem Idealismus wirft nicht zuletzt ein neues Licht auf sein philosophisches Verhältnis zur Religion. Als Sohn des Kantors der israelitischen Gemeinde von Coswig aufgewachsen, verfügte er über eine große religiöse Musikalität, und diese hat auch den Ton seiner philosophischen Texte geprägt, was bisweilen selbst bei wohlwollenden Interpreten Irritationen und Reserve auslöst. Und in der Tat war er kein methodischer Atheist, der sich in seinen philosophischen Überlegungen grundsätzlich von jeglichem Rückgriff auf Religion fernhielt. Allerdings ging es Cohen selbst in seinem religiösen Denken nicht einfach darum, religiöse Überzeugungen zu rechtfertigen, sondern sein Ziel war es, gewisse Aspekte der Religion auf ihren rationalen Sinn hin zu befragen und - soweit ein solcher erkennbar ist - für die philosophische Vernunft zu erschließen. Cohens Religionsphilosophie ist daher keine theologische Metaphysik, die sich der Philosophie als einer Magd bedient, sondern eine vernunftgeleitete Hermeneutik, welche religiöse Begriffe, Texte oder Gebote unter philosophischen Vorzeichen rekonstruiert.

Cohens Texte werden heute fast nur noch von Spezialisten gelesen. Das ist einerseits eine Folge davon, dass die Rezeption seiner Werke - sieht man von gewissen religionsphilosophischen Diskussionen ab - durch die beiden Weltkriege gewaltsam unterbrochen worden ist. Cohen selber erlebte das Ende des Ersten Weltkriegs nicht mehr, und er konnte sich nicht mehr in die Diskussion der Zwischenkriegszeit einschalten, was umso bedauerlicher ist, als er in seiner Religionsphilosophie, ausgehend vom Problem der Armut und dem sozialen Leid, eine Konzeption menschlicher Endlichkeit vorgelegt hat, die als valide systematische Alternative zu Heideggers Ansatz begriffen werden kann. ${ }^{13}$ Nach dem Zweiten Weltkrieg war Cohen im kulturellen Gedächtnis fast vollends in den Hintergrund gerückt. ${ }^{14}$

Dass Cohen heute fast nur noch von Spezialisten gelesen wird, hat andererseits aber auch mit der enormen Schwierigkeit seiner Werke zu tun. Viele seiner Aussagen sind ohne Kenntnis des gesamten Werkes nur schwer zu verstehen. Obwohl Cohen den Systembegriff erst auf Anraten des Verlegers und nicht ohne Widerstand auf seine eigene Philosophie angewendet hat ${ }^{15}$, ist er einem ausgesprochen systematischen Philosophieverständnis verpflichtet, dem zufolge zwischen Aussagen der Erkenntnistheorie, der Ethik- und Rechtsphilosophie, der Ästhetik sowie der Religionsphilosophie ein enger methodischer Zusammenhang besteht. Festlegungen auf bestimmte Positionen im Bereich der theoretischen Philosophie trifft er stets im Wissen um allfällige Implikationen für den Bereich der praktischen Philosophie, und umgekehrt; und selbst seine Ästhetik ist - und zwar bis in einzelne Werkinterpretationen hinein - geprägt von einem Sinn für die philosophischen Implikationen künstlerischer Aussagen und Entwicklungen. Allerdings macht Cohen solche Zusammenhänge oft nicht explizit, sondern er teilt sie bestenfalls durch seine ebenso reichhaltige wie pointierte Metaphorik mit. Das heißt aber nicht, dass seine Thesen unbegründet wären, sondern nur, dass man die Argumente dahinter oft erst mühsam und unter Einbezug anderer Texte rekonstruieren muss. „Man ist geneigt zu sagen: Der mos geometricus finde sich in seinem Denken zwischen den Zeilen“, hat Reiner Wiehl diesen Sachverhalt einmal umschrieben. ${ }^{16}$

Das vorliegende Heft soll den Lesern und Leserinnen einen ersten Zugang zu Cohen verschaffen, wobei nicht nur und nicht primär in die verschiedenen Aspekte von Cohens Denken

13 Siehe dazu auch U. Renz, Die Rationalität der Kultur. Kulturphilosophie und ihre transzendentale Begründung bei Cohen, Natorp und Cassirer, Hamburg 2002, $279 \mathrm{f}$.

14 Vgl. für eine differenziertere Analyse der Rezeptionsgeschichte auch das in diesem Heft abgedruckte Interview mit Myriam Bienenstock, Helmut Holzhey und Andrea Poma.

15 H. Holzhey, Einleitung des Herausgebers, in: H. Cohen, Werke, 6, VII.

16 R. Wiehl, Subjektivität und System, Frankfurt/M. 2000, 221. 
eingeführt werden soll, sondern gezeigt wird, inwiefern Cohens Denken auch heute noch befruchtend und relevant sein kann. Das entspricht durchaus der allgemeinen Tendenz der Cohen-Forschung: Zwar macht die Rekonstruktion seiner Überlegungen, wie schon ausgeführt, einen großen hermeneutischen Aufwand erforderlich. Trotzdem ist das Interesse an Cohen oft systematischer Natur: Seine Texte werden nicht zuletzt deshalb überhaupt noch gelesen, weil man in ihnen immer wieder auf Anregungen für unterschiedlichste systematische Problemstellungen stößt. Dabei tun sich Philosophen kontinentaler Prägung etwas leichter, doch es sind auch Zugänge möglich, die von Fragen ausgehen, wie sie in der analytischen und postanalytischen Philosophie diskutiert werden.

Am leichtesten zugänglich ist sicher Cohens praktische Philosophie. Andrea Esser, die sich seit ihrer eigenen Berufung nach Marburg mit Cohen beschäftigt, widmet sich in ihrem Beitrag dem Problem des Autonomiebegriffs. Ausgehend von einer Problematisierung gegenwärtiger Positionen, schlägt sie vor, Cohens Ethik für eine Neuformulierung des Autonomiebegriffs heranzuziehen. Dabei sind ihr zwei Momente besonders wichtig: zum einen das in der Ethik des reinen Willens skizzierte Wissenschaftsverständnis und zum anderen Cohens Begriff des moralischen Selbst. Wie Esser deutlich macht, begreift Cohen die Ethik in einem doppelten Sinne als ein wissenschaftliches Unterfangen: Sie ist auf das Faktum der Rechtswissenschaft bezogen, und sie versteht sich selbst als wissenschaftliche Praxis. Daraus resultiert ein Verständnis von Ethik, das, so Esser, eine Reduktion von Autonomie auf individuelle Selbstbestimmung von vorneherein ausschließt. Eine besondere Bedeutung weist Esser ferner Cohens ,am Paradigma der staatlichen Integration“ orientierten Begriff des moralischen Selbst zu, der einem individualistischen Reduktionismus bereits in den Grundbegriffen einen Riegel vorschiebt. Insgesamt liegt nach Esser der Gewinn von Cohens Ansatz darin, dass der Rede von der autonomen Selbstbestimmung ein Sinn abgewonnen werden kann, der sowohl individualistische als auch kollektivistische Einseitigkeiten vermeidet.

Auf Ähnlichkeiten mit Ansätzen der analytischen Philosophie weist der Beitrag der Gastherausgeberin hin, der einige Grundzüge von Cohens theoretischer Philosophie anhand eines Vergleichs des Marburger Neukantianismus mit den Ansätzen von Wilfrid Sellars und Robert Brandom erläutert. Wie der Beitrag zeigt, gibt es zahlreiche überraschende Übereinstimmungen, angefangen von der sowohl Cohens als auch Sellars' Kant-Interpretation zu Grunde liegenden These, dass Kant in der Kritik der reinen Vernunft einen neuen Begriff der Erfahrung entwickelt habe, über die Kritik am Mythos des Gegebenen, bis hin zum sowohl von Cohen als auch von Brandom unternommenen Versuch, eine Theorie des begrifflichen Gehalts zu entwickeln, die ohne den Repräsentationsbegriff auskommt.

Mit Blick auf einen anderen Hintergrund schlägt der Beitrag von Reinier Munk eine aktualisierende Lesart von Cohens Denken vor. Vor dem Hintergrund der vor allem von Emmanuel Lévinas geäußerten Kritik am totalitären oder gar gewaltsamen Charakter idealistischen Denkens veranschlagt Munk die Relevanz von Cohens Ansatz darin, dass er eine alternative Form des Idealismus zur Diskussion stelle, die sowohl von Kant als auch vom deutschen Idealismus in entscheidenden Punkten abweicht. Von Kant unterscheidet sich Cohens Idealismus nach Munk nicht nur darin, dass er auf die Zwei-Stämme-Lehre verzichtet, sondern auch darin, dass Cohens Idealismus eine Theorie des Denkens und nicht der Subjektivität entwickelt. Wie Munk auf der anderen Seite festhält, versteht Cohen seinen Idealismus dennoch als ein erkenntniskritisches Projekt, dessen zentrales Thema die Geltungsbedingungen wissenschaftlichen Wissens bildet. Nicht zuletzt sei entscheidend, dass Cohens Idealismus den dialogischdialektischen Charakter begrifflichen Denkens unterstreiche. Daher sei nicht erst in der Ethik, sondern bereits in der Logik das Problem des Anderen - wenn auch in unpersönlicher Form virulent. 
In den Kontext der gegenwärtig im Bereich der jüdischen Philosophie geführten Debatte über die Frage eines „Textual Reasoning“ stellt Pierfrancesco Fiorato das Denken Hermann Cohens. Zentrales Anliegen dieser Debatte ist die Auseinandersetzung mit einem an der jüdischen Hermeneutik orientierten Begriff der Vernunft, dessen paradigmatische Leistung nicht in der unmittelbaren Erkenntnis von Dingen, sondern in der kritischen Auslegung von Texten über die Dinge bestehen soll. Es geht also, so könnte man diese Debatte auch charakterisieren, um die Frage nach der Begründung eines alternativen und nicht auf die Annahme eines lumen naturale abgestützten Rationalismus. Wie Fiorato anhand einer subtilen Auslegung der Verhältnisbestimmungen von ungeschriebenem Gesetz und schriftlicher Lehre in der Ethik und in der Religionsphilosophie zeigt, kann Cohen durchaus als ein Vorläufer eines solchen Rationalismus verstanden werden.

Der Beitrag von Hartwig Wiedebach setzt sich anhand einer Darstellung von Cohens wissenschaftlicher Biographie mit dem Verhältnis von religiösem Glauben und philosophischem Denken auseinander. Den Hintergrund bildet die die Cohen-Rezeption jahrzehntelang bestimmende Auffassung Franz Rosenzweigs, wonach das religionsphilosophische Spätwerk Religion der Vernunft aus den Quellen des Judentums als Ausdruck einer Heimkehr zur Religion seiner Väter zu begreifen sei. ${ }^{17}$ Diese Auffassung gilt in der Cohen-Forschung schon seit längerem als überwunden ${ }^{18}$, doch damit gewinnt das Problem, wie man das Verhältnis von Cohens jüdischem Glauben zu seiner Philosophie verstehen kann, erst recht an Gewicht. Und dieses Problem ist umso virulenter, als es in einem engen Zusammenhang mit der Frage nach einer angemessenen Interpretation von Cohens philosophischer Entwicklung steht. Wie Hartwig Wiedebach in seinem Beitrag zeigt, gibt es durchaus verschiedene Motive von Cohens Denken, die einerseits in verschiedenen Stadien der Entwicklung seines Denkens eine Rolle gespielt haben, andererseits für das Verständnis der Beziehung von jüdischem Glauben und Philosophie aufschlussreich sind. Zu nennen ist hier zum Beispiel das Verhältnis von Sprache und Physiologie, das sowohl in den frühen sprachpsychologischen Schriften als auch in der Ästhetik des reinen Gefühls sowie schließlich in mehreren religionsphilosophischen Texten eine wichtige Rolle spielt, oder die Bedeutung der Metapher des Herzens und damit das Problem der Affektivität, die sowohl in der Ethik Cohens als auch in der Religion der Vernunft unterstrichen wird. Diese Motive geraten allerdings, wie Hartwig Wiedebach argumentiert, überhaupt erst in den Blick, wenn man Cohens Philosophie nicht einfach unter der Kategorie ,Neukantianismus' ablegt.

Ernst Cassirer hat seine Rede am Grab von Hermann Cohen mit den Worten eröffnet: „Nicht von der Leistung Hermann Cohens - nicht von dem, was er als Denker und Forscher der Philosophie gegeben hat, kann ich in dieser Stunde zu sprechen versuchen. Das Urteil hierüber gehört der Geschichte an - und, je allgemeiner die Kenntnis, je tiefer das Verständnis der Werke im Laufe der Geschichte werden wird, um so bestimmter und unzweideutiger wird ihr Urteil über die Gesamtheit seines Wirkens lauten. "' ${ }^{19}$ Ein solches Urteil ist bis heute

17 F. Rosenzweig, Einleitung, in: H. Cohen, Jüdische Schriften, Bd. 1, XIII-LXIV; sowie auch in: F. Rosenzweig, Gesammelte Schriften, III: Zweistromland. Kleinere Schriften zu Glauben und Denken, Dordrecht 1984, 177-223.

18 Siehe auch H. Holzhey, Vorwort, in: Religion der Vernunft aus den Quellen des Judentums. Tradition und Ursprungsdenken in Hermann Cohens Spätwerk. Religion of reason out of sources of Judaism - tradition and the concept of origin in Hermann Cohen's later work, hg. v. H. Holzhey u. a., Hildesheim 2000, X.

19 E. Cassirer, Hermann Cohen. Worte gesprochen an seinem Grabe am 7. April 1918, in: Neue Jüdische Monatshefte, 2 (1918), 347-352, 347. 
ausgeblieben. Es wird auch nicht mehr gelingen, die Bedingungen herbeizuführen, die die Nachwelt befähigt, dieses Urteil zu fällen. Man mag das bedauern oder sogar beklagen. Doch nicht um Hermann Cohen gerecht zu werden, sollten wir seine Texte lesen. Sondern weil und sofern sich dies aus der Sicht heutiger Problemstellungen lohnt. Vielleicht gehen wir mit seiner Philosophie daher am besten so um, wie Cohen mit der Tradition umgegangen ist: in einer Haltung der Treue und der Kritik.

Ursula Renz, Klagenfurt 